\title{
EFFECTIVENESS AND SAFETY OF PEMBROLIZUMAB MONOTHERAPY IN PATIENTS WITH LOCALLY ADVANCED OR METASTATIC NON-SMALL CELL LUNG CANCER
}

\author{
Rocio Tamayo-Bermejo ${ }^{1}$, Juan Carlos Del Rio-Valencia ${ }^{1}$, Beatriz Mora Rodríguez ${ }^{2}$, and \\ Casillo-Muñoz Isabel ${ }^{1}$ \\ ${ }^{1}$ Hospital Regional Universitario de Malaga \\ ${ }^{2}$ Hospital Regional Universitario Carlos Haya
}

September 8, 2020

\begin{abstract}
ABSTRACT Introduction: Immunotherapy has become a standard treatment for lung cancer; the objective of this study was to evaluate the effectiveness, safety of pembrolizumab monotherapy in patients with advanced or metastatic Non-Small Cell Lung Cancer (NSCLC) used in real-world clinical practice. Material and methods: Retrospective observational study of every patients treated with pembrolizumab in our centre from January 2017 to June 2019. Outcomes collected: sex, age, ECOG, PDL-1 levels, previous metastatic line therapies, adverse events (AE) and smoking status. Results: A total of 62 patients were reviewed. The median age was $62.34 \pm 10.62$ years, $48(77.41 \%)$ were men and $91.93 \%$ of patients had ECOG 0 . The median doses administered was $170.5 \mathrm{mg}(108-240 \mathrm{mg}$ ) and median follow-up was 3 months (range: 1-38). A median of 4 cycles of pembrolizumab (range 1 to 56) were administered as monotherapy. The reason for treatment discontinuation was mainly due to disease progression in $38.70 \%$ patients or death in $30.64 \%$. As first-line pembrolizumab monotherapy, median progression free survival was 7.7 months (95\% CI: 3.66-11.73) (N=33). With respect to patients who were treated in second-thirdline treatment, median PFS was 3.5 months (95\%CI: 2.40-4.59) ( $\mathrm{N}=29)$. As to overall survival, pembrolizumab-treated patients as first-line treatment reached 19 months median OG (95\% CI: 13.36-24.63) (N=33) and those treated in second-third-line treatment got 11 months (95\% CI: 3.4-18.5). 64.51\% of patients presented some AE to pembrolizumab however, only, $9.38 \%$ of them were grade 3. Conclusion: Pembrolizumab represents an effective and feasible alternative in terms of SLP. It is a well-tolerated treatment option.
\end{abstract}

\section{Hosted file}

main article (1).pdf available at https://authorea.com/users/357027/articles/479800effectiveness-and-safety-of-pembrolizumab-monotherapy-in-patients-with-locally-advancedor-metastatic-non-small-cell-lung-cancer 


\begin{tabular}{|c|c|c|c|c|}
\hline Characteristics & $\begin{array}{l}\text { Total } \\
(\mathrm{n}=62)\end{array}$ & $\begin{array}{l}\text { First line } \\
(n=33)\end{array}$ & $\begin{array}{l}\text { Second line } \\
(n=26)\end{array}$ & $\begin{array}{l}\text { Third line } \\
(n=3)\end{array}$ \\
\hline \multicolumn{5}{|l|}{ Age (years) } \\
\hline - Median & 64 & 64 & 64 & 69 \\
\hline - Rank & $39-86$ & $40-75$ & $39-86$ & $55-78$ \\
\hline \multicolumn{5}{|c|}{ - Age (category) - n (\%) } \\
\hline$<65$ years & $33(53.23 \%)$ & $18(54.55 \%)$ & $12(46.15 \%)$ & $1(33.33 \%)$ \\
\hline$\geq 65-75$ & $22(35.48 \%)$ & $11(33.33 \%)$ & \begin{tabular}{|l|l|}
$10(38.46 \%)$ \\
\end{tabular} & 1 (33.33\%) \\
\hline$\geq 75$ & $7(11.29 \%)$ & $2(6.06 \%)$ & $4(15.38 \%)$ & $1(33.33 \%)$ \\
\hline \multicolumn{5}{|l|}{ Sex - n (\%) } \\
\hline Men & $48(77.42 \%)$ & $26(78.79 \%)$ & 19 (73.08\%) & $3(100 \%)$ \\
\hline Women & $14(22.58 \%)$ & $7(21.21 \%)$ & $7(26.92 \%)$ & 0 \\
\hline \multicolumn{5}{|l|}{ ECOG - n (\%) } \\
\hline 0 & $19(30.65 \%)$ & $10(30.30 \%)$ & $8(30.77 \%)$ & $1(33.33 \%)$ \\
\hline 1 & $37(59.68 \%)$ & $19(57.58 \%)$ & $16(61.54 \%)$ & $2(66.67 \%)$ \\
\hline$\geq 2$ & $4(6.45 \%)$ & $3(9.09 \%)$ & $1(3.85 \%)$ & - \\
\hline Not collected & $2(3.23 \%)$ & $1(3.03 \%)$ & $1(3.85 \%)$ & - \\
\hline $\begin{array}{l}\text { CNS†Metastase } \\
\mathrm{s}-\mathrm{n}(\%)\end{array}$ & $16(25.81 \%)$ & $11(33.33 \%)$ & $4(15.38 \%)$ & $1(33.33 \%)$ \\
\hline \multicolumn{5}{|c|}{ Previous treatment - $n(\%)$} \\
\hline $\begin{array}{l}\text { Carboplatin- } \\
\text { gemcitabine }\end{array}$ & $10(16.13 \%)$ & - & $9(34.62 \%)$ & $1(33.33 \%)$ \\
\hline Carboplatin-taxol & $8(12.90 \%)$ & - & 7 (26.92\%) & $1(33.33 \%)$ \\
\hline $\begin{array}{l}\text { Carboplatin- } \\
\text { pemetrexed }\end{array}$ & $11(17.74 \%)$ & - & $10(38.46 \%)$ & $1(33.33 \%)$ \\
\hline $\begin{array}{l}\text { Cisplatin- } \\
\text { vinorelbine }\end{array}$ & $2(3.23 \%)$ & - & & $2(66.67 \%)$ \\
\hline \multicolumn{5}{|c|}{ Tumour Histological features - $\mathbf{n}(\%)$} \\
\hline Squamous & $25(40.32 \%)$ & $13(39.39 \%)$ & 11 & $1(33.33 \%)$ \\
\hline Non-squamous & $35(56.45 \%)$ & $20(60.61 \%)$ & $13(50 \%)$ & $2(66.67 \%)$ \\
\hline Unknown & $2(3.23 \%)$ & - & $2(7.69 \%)$ & - \\
\hline \multicolumn{5}{|c|}{ Smoking status - $\mathrm{n}(\%)$} \\
\hline Smokers & $55(88.71 \%)$ & $29(87.88 \%)$ & $23(88.46 \%)$ & $3(100 \%)$ \\
\hline Non-smokers & $7(11.29 \%)$ & $4(12.12 \%)$ & $3(11.54 \%)$ & - \\
\hline \multicolumn{5}{|c|}{ PD-L1 level (\%)- n (\%) } \\
\hline$(\geq 50 \%)$ & $52(83.87 \%)$ & $31(9.94 \%)$ & 19 (73.08\%) & $2(66.67 \%)$ \\
\hline
\end{tabular}

